Cad. Est. Ling., Campinas, 46(1):91-109, Jan./Jun. 2004

\title{
POUR UNE ADAPTATION DU MODÈLE EUROPÉEN D'ENSEIGNEMENT DES LANGUES ÉTRANGÈRES EN CONTEXTES LATINO-AMÉRICAINS ${ }^{1}$
}

\author{
SAMANTHA CHAREILLE \\ (Alianza Colombo Francesa de Bogotá)
}

\begin{abstract}
RESUMEN La mayoría de los estudios franceses que se consagran a la investigación sobre autonomía en el ámbito de las lenguas extranjeras se refiere a los países europeos pero más escasos son los que hacen referencia a América latina. Porque precisamente tuvimos que llevar a cabo investigaciones similares sobre, entre otros, la planificación lingüística del Mercado común del Cono sur y de Chile (2001), el estatuto del francés en Uruguay (1997 y 2003) y en Colombia (2002), estuvimos especialmente interesadas por el tema de la enseñanza de las lenguas en América latina y por los sistemas educativos formales de la zona.

Nuestro objetivo será poner de manifiesto que algunos principios de las políticas lingüísticas educativas europeas podrían ser utilizables en los contextos latinoamericanos. Nos concentraremos en particular en el Marco europeo común de referencia para las lenguas, su Portafolio y en el modelo europeo de centro de recursos de lenguas. También se hará referencia a tales conceptos como las habilidades lingüísticas parciales y la autonomía. El marco limitado de este artículo, que no nos permite ser tan exhaustivas como lo desearíamos, comenzaremos por un rápido panorama de la situación educativa latinoamericana con un énfasis en la enseñanza de las lenguas. Luego pasaremos a la exposición de las políticas lingüísticas educativas europeas que nos interesan en el marco de este estudio para concluir sobre los centros de recursos en cuestión.
\end{abstract}

La plupart des études françaises qui sont consacrées à la recherche sur l'autonomie dans le domaine des langues étrangères porte sur les pays européens mais plus rares sont celles portant sur l'Amérique latine. Parce que nous avons justement été amenée à mener des recherches approchantes sur, entre autres, l'aménagement linguistique du Marché commun du Cône sud et du Chili (2001), le statut du français en Uruguay (1997 et 2003) et en Colombie (2002), nous avons été particulièrement intéressée par le thème de l'enseignement des langues en Amérique latine et donc par les systèmes éducatifs formels de la zone.

Notre propos sera de montrer que certains principes des politiques linguistiques éducatives européennes pourraient être utilisables en contextes latino-américains. Nous nous concentrerons en particulier sur le Cadre européen commun de référence pour les langues (C.E.C.R.) son Portfolio (P.E.L.) et sur le modèle européen de centre de ressources de langues. Des notions telles que celles de compétences langagières partielles et autonomie sont également régulièrement évoquées. Le cadre restreint de cet article ne nous permettant pas d'être aussi exhaustive que nous le souhaiterions, nous commencerons par un rapide

\footnotetext{
${ }^{1}$ Propos du psychologue Herbert GerJuoy cité par Alvin Toffler dans le Choc du Futur, Folios Essais,
} 1987, Paris 
CHAREILLE - Pour une adaptation du modéle...

panorama brossé à grands traits de la situation éducative latino-américaine avec une centration évidente sur l'enseignement des langues puis nous passerons à l'exposé des politiques linguistiques éducatives européennes nous intéressant dans le cadre de cette étude pour conclure sur les centres de ressources en question.

\section{SURVOL DE LA SITUATION ÉDUCATIVE ET DE L'ENSEIGNEMENT DES LANGUES EN AMÉRIQUE LATINE.}

Quelle que soit leur nationalité, les systèmes scolaires modernes ont souvent eu comme finalité celle d'arriver à une certaine homogénéisation en matière de langage, de valeurs, d'intériorisation des normes et de connaissance de la population. Ils constituent en même temps l'outil le plus utilisé et le plus efficace employé par les gouvernements afin d'implanter leurs politiques linguistiques (Samantha Chareille, 2001).

En Amérique latine, depuis trente ans, l'école s'est démocratisée et, de nos jours, le taux d'alphabétisation a considérablement augmenté. Elle s'est modernisée et les programmes de langue ont été modifiés pour donner la parole aux élèves et tenir davantage compte de l'expérience individuelle. La formation des enseignants a été améliorée et les exigences ont considérablement augmenté. La recherche sur l'enseignement s'est, elle aussi, beaucoup développée. Il n'en demeure pas moins que la situation globale est perçue par une grande partie de la population comme insatisfaisante, notamment en matière d'enseignement linguistique.

La majorité des systèmes de l'enseignement secondaire latino-américaines inclue l'enseignement d'au moins une langue étrangère obligatoire - l'anglais - voire d'autres langues optionnelles. On peut donc dire qu'à mesure que s'est effacée la séparation entre l'enseignement primaire et le secondaire et qu'a augmenté la durée de la scolarité obligatoire, l'enseignement des langues étrangères s'est peu à peu étendu à toute la population. Il s'agit là bien évidemment d'un progrès et il convient de l'évaluer positivement. Il est cependant tout aussi évident que nous sommes actuellement loin des résultats désirés.

On constate en effet que la majorité des systèmes éducatifs est souvent trop fragile et pauvre pour maintenir l'enseignement obligatoire de plusieurs langues et opte généralement pour l'anglais. A se demander si le statut socio-symbolique des langues trouve à l'école une consécration ou une remise en question...

Ce phénomène peut s'expliquer par deux types de facteurs : internes (rendement scolaire, aspect financier, problème de formation, etc.) et externes (politiques, économiques et sociaux). 
Cadernos de Estudos Lingüísticos 46(1) - Jan./Jun. 2004

\subsection{Facteurs internes: la question des ressources financières limitées.}

Lors du symposium «Education et formation : entre l'incontournable compétition et l'indispensable égalité des chances » (19.09.1997), María DE IBARROLA² a souligné que l'égalité en matière de pédagogie était loin d'être acquise sur le continent américain, malgré les efforts importants déployés en ce sens. Il existe bien une apparence d'égalité d'accès à l'éducation primaire, par contre, le tableau s'assombrit dès que l'on aborde la fréquentation de l'école secondaire pour devenir catastrophique en matière d'enseignement supérieur. Parallèlement, trop d'étudiants entrent à l'université et y demeurent durant des années, sans avoir les aptitudes ni le goût de réussir. Qui plus est, la prolifération d'institutions privées n'a jamais été accompagnée de processus d'évaluation rigoureux et transparents de la qualité de l'enseignement. Ce constat est corroboré par une étude effectuée par le directeur du bureau régional de l'U.N.E.S.C.O. pour l'Amérique latine et par l'U.N.I.C.E.F. dans une enquête internationale - La situation des enfants dans le monde 1999 - qui estime que le rendement des systèmes scolaires de base des pays latino-américains est insuffisant. Résultat, si les taux de scolarisation n'ont jamais été aussi élevés, les ratés du système scolaire se soldent par une énorme proportion d'analphabètes fonctionnels (près d'un milliard de personnes), c'est-à-dire des personnes ne sachant ni lire ni même écrire leur nom. Etant donné le rapport direct entre la lecture et les divers niveaux de développement, un tel résultat est désastreux.

Selon l'U.N.I.C.E.F., c'est en Amérique latine que les disparités économiques sont les plus prononcées entre riches et pauvres. Parallèlement, les méthodes d'enseignement tendent à être rigides et traditionnelles, ce qui décourage les élèves de rester à l'école.

Il est difficile de trouver les raisons d'une situation aussi complexe y compris dans les enquêtes élaborées par des organismes tels que la C.E.P.A.L. et les divers instituts de statistiques des pays de la zone. Les problèmes auxquels sont confrontés les systèmes éducatifs de la région ne peuvent pas être séparés de ceux vécus par les sociétés latinoaméricaines qui, depuis les années cinquante jusqu'à aujourd'hui, ont connu une crise des modèles sociaux, des confrontations, une reconversion, une étape de réajustement économique et une difficile réinitiation à la démocratie. Les régimes militaires, surtout, ont pour beaucoup contribué à une désintégration profonde de ces systèmes en procédant à des purges massives dans le milieu enseignant, substituant à ces derniers un personnel acquis à leur cause, réduisant les budgets affectés à l'éducation et fondant leur enseignement sur la peur, la sanction, la délation et le rejet du monde extérieur. Le retour à la démocratie n'a pas permis de se débarrasser de ce lourd héritage. "L'éducation argentine a été un facteur de modernisation et de démocratisation de notre société [...] Mais malheureusement depuis assez longtemps déjà, elle a cessé de l'être ». Cette réflexion d'Aldo NERI ${ }^{3}$ prouve

${ }^{2}$ Directrice de la Fondation du Syndicat national des travailleurs de l'éducation du Mexique pour la culture des enseignants depuis 1993.

${ }^{3}$ Ancien Ministre et député de l'Union Centriste Radicale, parti de l'ex-président Alfonsín, premier président du retour démocratique. Réflexion développée dans le journal La Nación le 12 février 1988. 
CHAREILLE - Pour une adaptation du modéle...

l'importance de la prise de conscience, au sein des classes dirigeantes de la détérioration sensible et continue des conditions d'enseignement. Malheureusement, l'incapacité des démocraties à gérer les situations de crise et les projets des systèmes éducatifs a fini par scléroser ce dernier.

L'éducation est un investissement dans les qualifications humaines qui peut contribuer à promouvoir la croissance économique, à accroître la productivité, à favoriser l'épanouissement personnel et le développement social et réduire les inégalités sociales. Comme tout investissement, il a des coûts, une rentabilité et des pertes. Aucune équation ne permet de décrire parfaitement ces interactions, mais certains indicateurs permettent de mieux comprendre le coût et la nature de l'investissement dans l'éducation ainsi que les bénéfices ou le rendement de ce dernier.

L'explosion en éducation, qui s'est produite en moins d'une dizaine d'années, s'est faite sans l'existence de conditions matérielles, de concepts éducatifs et de ressources humaines nécessaires. L'augmentation du nombre d'élèves a généré une chute spectaculaire de la qualité de l'enseignement au moment précis où la crise de l'endettement international des pays de la zone a entraîné d'importants programmes de réajustement fiscal. Conséquence directe : la disponibilité des ressources par élève a diminué et ce malgré les souhaits exprimés par les dirigeants. Les limitations financières ont beaucoup joué sur la qualité de l'enseignement à travers la restriction des investissements effectués dans les infrastructures et les équipements qui n'ont donc pas pu accompagner l'augmentation du nombre d'inscrits.

Aujourd'hui plus que jamais, les limitations des ressources financières ont un impact important sur la qualité du corps enseignant à travers des salaires de plus en plus médiocres. (C.E.P.A.L., 1995). Après avoir maintenu près de vingt ans le développement humain en arrière de la mondialisation et de l'ajustement structurel, peut-être allons-nous voir s'ouvrir l'ère de l'investissement dans « le capital humain et social » qui facilitera considérablement la diffusion dans le monde entier de la révolution de l'éducation, espère le rapport $L a$ situation des enfants dans le monde 1999. Aujourd'hui, les plus éminents économistes, y compris ceux qui travaillent au F.M.I. et à la Banque mondiale, considèrent que la privatisation n'est pas la seule clef de la croissance économique à long terme. Le capital humain (la santé, l'éducation et la nutrition de la nation) et le capital social (la culture, les valeurs partagées et une société civile vigoureuse) sont tout aussi essentiels.

\subsection{Facteurs externes.}

\subsubsection{Dimension politique.}

Lorsqu'il existe en matière linguistique une politique volontariste, l'école est le lieu prioritaire des réformes et des expérimentations et elle joue un rôle d'accélérateur du changement. Inversement, quand la politique décidée est une politique du « laisser-faire », le microcosme scolaire, qui relaie la société, joue un rôle conservateur et le statut des langues ne fait que s'y conforter. Or, dans l'ensemble des pays de la région sur laquelle porte notre étude, la dimension politique de l'enseignement des langues étrangères n'est 
Cadernos de Estudos Lingüísticos 46(1) - Jan./Jun. 2004

pas encore traitée de façon adéquate face aux grands changements qui se vérifient dans le domaine de l'éducation (cf. Samantha ChAREILLE, 2001 pour le cas des pays du Mercosur ${ }^{4}$ et du Chili).

\subsubsection{Dimension économique.}

Le contexte de mondialisation actuel se pense de plus en plus en termes de marché ce qui n'a bien sûr pas échappé aux domaines de l'enseignement-apprentissage des langues : références de plus en plus fréquentes aux notions d'offre, de demande, de consommateurs, etc. En dépit de certaines résistances, les langues sont désormais des biens capitalisables et négociables. Cette conception «économique » des langues et de leur enseignement a longtemps été absente des politiques éducatives des pays de la région, exception faite, peut-être, de celles touchant à l'anglais. Depuis la création de regroupements régionaux tels que le Mercosur, une approche économique a heureusement provoqué quelques changements si ce n'est pratiques, tout du moins théoriques. Soulignons toutefois que l'exclusion encore trop fréquente de la question des langues étrangères des débats sur les nouvelles tendances de l'éducation écarte les possibilités d'intégration réelle de cet enseignement dans le contexte éducatif actuel.

\subsubsection{Dimension sociale.}

Aucune opération de formation en langue étrangère ne peut faire abstraction de la prise en compte des usages sociaux de la langue cible dans la communauté dont elle est issue. De même, il n'est pas possible d'ignorer les usages linguistiques en vigueur dans la société où se déroule le processus d'enseignement-apprentissage. Nous entendons par usages sociaux les statuts (attribués par les hommes aux langues), les situations d'emploi et les fonctions. En effet, les parlers dont le statut est «inférieur », non officiel, ne sont généralement pas considérés comme des objets d'enseignement potentiels (cf. langues minorées). Il existe également un statut scolaire des langues jouant pour beaucoup dans leur insertion dans les systèmes scolaires formels d'où la distinction langue enseignée dans le primaire, le secondaire et le supérieur. A ces usages sociaux, il convient également d'ajouter le niveau d'acceptation ou de diffusion de la langue cible, c'est-à-dire le statut informel. On range généralement dans cette catégorie les notions d'utilité, de facilité, de prestige de sympathie, etc.

Ces phénomènes jouent un rôle fondamental au sein de l'ensemble des facteurs qui interviennent dans le choix des langues à l'école. En effet, on imagine mal légitimer et enseigner un parler si celui-ci n'est pas reconnu et n'a aucune chance d'être adopté par le groupe social il est destiné. Dans ce contexte les médias jouent un rôle décisif (Samantha ChAREILLE, 2001, chapitre VI). Louise DABÈnE (1994, p. 53) souligne à ce titre que si c'est

\footnotetext{
${ }^{4}$ Marché commun du Cône sud, créé par le Traité d'Asunción de mars 1991, et comprenant l'Argentine, le Brésil, le Paraguay et l'Uruguay.
} 
CHAREILLE - Pour une adaptation du modéle...

généralement l'appréciation de l'utilité de la langue qui détermine le choix des parents pour la première langue étrangère, lorsqu'il y a possibilité d'en choisir une seconde, ces derniers sont surtout guidés par le coefficient de sympathie et la représentation de difficulté de l'apprentissage. Ce n'est pourtant pas en vertu de quelques dispositions naturelles qu'une langue s'impose dans des domaines prestigieux comme le commerce, les sciences ou la musique. Par exemple, rien dans le système linguistique de l'anglais ne le prédispose à dominer les affaires ou les sciences.

Un autre problème que l'on soulève souvent: le degré de difficulté d'une langue. On semble accepter généralement l'idée que certaines langues sont plus difficiles ou plus faciles à apprendre que d'autres. Ainsi, nombreux sont ceux qui croient que l'anglais doit à sa prétendue facilité d'apprentissage son pouvoir d'attraction à l'heure actuelle. Le degré de difficulté d'une langue demeure toujours une question très discutable et arbitraire, parce que l'on doit se placer du point de vue de la personne qui l'apprend comme langue seconde. Les difficultés d'apprentissage dépendent d'un ensemble de facteurs relativement complexes. Abstraction faite de tout contexte sociologique, un hispanophone devrait apprendre assez facilement les langues suivantes : l'italien, le français, le roumain, le portugais, l'indonésien (ou malais), le turc. Bien que ces langues soient techniquement faciles pour un hispanophone en raison d'affinités ou de compatibilités typologiques, c'est presque systématiquement l'anglais, plus difficile, qui est appris, situation se retrouvant chez les lusophones de la région. De même, le choix d'enseigner telle ou telle variété d'une langue (ríoplatense ou espagnol d'Espagne, par exemple) que nous avons amplement traité dans le chapitre précédent fait également l'objet de discussions sans fin.

En conclusion, on peut donc dire que, même si nous ne vérifions pas cela dans tous les établissements secondaires de la région et même si les réseaux officiels de quelques pays ont conservé l'enseignement de plus d'une langue étrangère, un pragmatisme évident marque l'enseignement des langues. En effet, les écoles d'élite qui gardent deux langues étrangères, généralement l'anglais et le français, ont deux sortes d'arguments : d'un côté la justification pratique de l'importance de l'anglais pour le monde moderne et la reconnaissance de l'hégémonie culturelle américaine et, de l'autre, essentiellement culturelle, l'importance de la culture française dû à la grande influence de cette dernière dans la plupart des systèmes éducatifs locaux. C'est pour ces raisons que la critique actuelle de l'école est que ce qu'elle enseigne (notamment dans le domaine des langues étrangères) sert une idéologie qui ne correspond pas toujours aux besoins de tous et qui est souvent fondée sur des critères d'appréciation qualitative qui ne devraient pas être appliqués aux langues (une tendance à classer, comparer, opposer et donc hiérarchiser les langues comme on le fait des peuples et des individus qui les composent). Aucun argument ne parle de l'importance de l'enseignement pluriel des langues étrangères pour le processus de libération des pays des influences étrangères et, par conséquent, pour la lutte en faveur de son autonomie culturelle.

C'est précisément ce constat qui nous amène à penser qu'une adaptation des principes européens d'enseignement des langues aux contextes latino-américains - principes fondés 
Cadernos de Estudos Lingüísticos 46(1) - Jan./Jun. 2004

sur la protection et le développement du patrimoine linguistique et la diversité culturelle en tant que source d'enrichissement mutuel - pourrait se poser en début de solution. Afin d'envisager cette possibilité, penchons-nous donc sur la situation européenne.

\section{ELÉMENTS DE POLITIQUES LINGUISTIQUES ÉDUCATIVES EUROPÉENNES TRANSPOSABLES EN CONTEXTES LATINO- AMÉRICAINS.}

\subsection{Le Conseil de l'Europe.}

Le Conseil de l'Europe est une organisation internationale (indépendante de la structure organique européenne) dont le siège est à Strasbourg et qui rassemble quarante cinq Etats démocratiques de l'Europe. Tout Etat européen peut devenir membre du Conseil de l'Europe à condition qu'il accepte le principe de la prééminence du droit. Il doit en outre garantir la jouissance des droits de l'homme et des libertés fondamentales à toute personne placée sous sa juridiction.

L'organisation a une vocation plurielle et traite toutes les grandes questions de la société européenne, à l'exception des problèmes de défense. Son programme de travail couvre, entre autres, les domaines suivants : droits de l'homme, médias, coopération juridique, cohésion sociale, santé, éducation, culture, patrimoine, sport, jeunesse, démocratie locale et coopération transfrontalière, environnement et aménagement du territoire.

Elle attache une importance particulière à la diversité du patrimoine linguistique et culturel de ses Etats membres. Ses activités dans le domaine des langues vivantes visent à favoriser le plurilinguisme et le pluriculturalisme des citoyens afin d'améliorer la communication et la compréhension mutuelle entre les personnes, et de lutter ainsi contre l'intolérance et la xénophobie. L'Europe est et restera multilingue ; face à cette réalité, le Conseil de l'Europe a élaboré au fil des ans une politique linguistique à travers ses projets « Langues vivantes » à Strasbourg que nous nous proposons maintenant d'examiner.

\subsection{Politique linguistique du Conseil de l'Europe.}

Le Conseil de l'Europe mène des activités dans le domaine des langues depuis une quarantaine d'années. Ses programmes sont coordonnés par deux instances complémentaires : la Division des politiques linguistiques à Strasbourg et le Centre européen pour les langues vivantes à Graz, créé il y a quelques années.

Le premier objectif du Conseil de l'Europe en matière de langues est de protéger et développer le patrimoine linguistique et la diversité culturelle de l'Europe en tant que source d'enrichissement mutuel en aidant ainsi chaque citoyen à comprendre, respecter et accepter autrui dans ses modes de pensées, ses sentiments, ses valeurs, ses croyances et ses traditions. Pour encourager ce genre d'attitude, le Conseil de l'Europe a élaboré une 
CHAREILLE - Pour une adaptation du modéle...

Charte européenne des langues régionales ou minoritaires. La Convention Cadre pour la protection des minorités nationales contribue par ailleurs à promouvoir ces objectifs.

Il souhaite également faciliter la mobilité des personnes et l'échange des idées en encourageant le développement de compétences communicatives dans une variété de langues, pour ainsi permettre aux uns et aux autres de coopérer plus efficacement.

Finalement il se donne pour but de développer une conception de l'enseignement des langues harmonieuse reposant sur des principes communs grâce à une mise en commun, par le biais de la coopération internationale, de l'expérience et des compétences des pays membres en ce domaine. L'idée est d'en arriver ainsi à une conception cohérente de l'enseignement des langues centré sur l'apprenant qui prenne en compte les buts et les objectifs de cet enseignement, son contenu, les expériences d'apprentissage et l'évaluation. Cela est facilité par des instruments tels que le Cadre européen commun de référence et le Portfolio européen des langues (qui nous semblent être aisément transposables en Amérique latine) dont l'une des particularités est de viser l'acquisition de différentes compétences incomplètes, c'est-à-dire des compétences langagières partielles.

Ce dernier aspect est particulièrement intéressant pour le cas latino-américain puisque, on l'a vu, les langues ne disposent, au sein des systèmes éducatifs en présence que d'un temps et d'un prestige limités. Cette situation étant peu susceptible de changer, pour le moment et dans certains pays tout du moins, peut-être serait-il souhaitable d'envisager d'autres objectifs d'apprentissage. A ce titre, nous pensons en particulier au développement de ce qui serait une compétence de compréhension qui permettrait, par exemple, aux communautés lusophones et hispanophones de communiquer dans leur propre langue.

Rappelons que le thème de l'intercompréhension des langues néolatines s'inscrit dans le thème plus général des politiques linguistiques. Il se fonde sur les études que mènent depuis déjà quelques années plusieurs universités européennes, centrées sur le problème de l'enseignement-apprentissage de l'intercompréhension entre des locuteurs de langues voisines ou de la même famille, dans notre cas, entre locuteurs de langues romanes. L'idée de comprendre les langues sans les parler se répand de plus en plus en Europe et atteint des cercles de plus en plus importants.

Les principes théoriques qui orientent ce travail correspondent à ceux du constructivisme appliqué à la compréhension des textes. Ici, c'est le lecteur lui-même qui construit le sens du texte d'après les associations qu'il établit entre ce que l'écrit dit et son expérience personnelle comprise comme la convergence de son système de connaissances, croyances et attitudes avec les circonstances contextuelles qui déterminent son entourage, au moment même de l'acte de lecture.

Ceci se traduit par une suite d'associations, évocations, assimilations, déductions et inférences qui permettent au lecteur de construire un sens, sens qui s'approche de celui que l'auteur a créé, mais qui conservera toujours son caractère personnel, vu que le 
Cadernos de Estudos Lingüísticos 46(1) - Jan./Jun. 2004

processus de la lecture est centré sur la personne, donc individuel. En effet, d'après certains théoriciens de la compréhension de lecture tels que Francine CicuRel, Gérard Vigner et Jean HÉBRARD, lire ne signifie pas seulement décoder, il s'agit d'une démarche interactive dans laquelle l'expérience personnelle du lecteur lui permet de hasarder des hypothèses. L'acte de lire résulte de deux séries de processus qui bien que de nature différente, aboutissent à la même finalité : la compréhension. D'une part le texte se présente comme un objet fait de signes graphiques, sorte d'indices, de pistes pour reconnaître un sens qui se complète de l'autre part, avec les connaissances préalables emmagasinées par le lecteur, ses capacités intellectuelles de déduction et autres qui lui permettent de construire un contexte cohérent et logique.

Or, les langues néolatines ne sont pas une exclusivité européenne. En Amérique latine se trouvent l'espagnol dans dix-neuf pays, le portugais au Brésil et le français dans deux Etats, Haïti et Québec. Citons à ce titre le projet d'investigation-action sur l'enseignementapprentissage de l'intercompréhension de langues latines chilien Lingua Sur dont le but est l'élaboration d'une méthodologie d'enseignement rapide de la compréhension en français pour apprenants hispanophones.

La coordination du projet a été prise en charge par le Service Linguistique et Culturel de l'Ambassade de France à Santiago du Chili et s'est associée au moyen d'une convention à trois universités nationales possédant un département de français langue étrangère et visant à la formation de maîtres : Université de Concepción, Université Métropolitaine de Sciences de l'Education de Santiago et Université de Playa Ancha de Valparaíso. L'expérience s'est articulée avec la recherche menée par le centre de Didactique des Langues de l'Université Stendhal Grenoble III, dans le cadre de Galatea.

En ce qui concerne les résultats il faut reconnaître que les distances entre les différentes régions ont empêché une cohésion de l'équipe, une coordination efficace, ce qui s'est répercuté dans l'application des tests. Cependant un échantillon représentatif de Santiago permet de signaler que $42 \%$ des élèves reconnaissent le thème principal, sont capables de saisir l'approche du texte et d'en déterminer le sens évoqué.

\subsection{Cadre européen commun de référence pour les langues.}

Le Conseil de l'Europe s'attache depuis longtemps à développer des consensus sur les objectifs et les principes d'orientation pour l'enseignement des langues. Grâce à ses programmes d'activités et ses publications, il poursuit l'adaptation de l'enseignement des langues aux besoins du monde contemporain. Dans cette tradition et parmi ses initiatives les plus récentes figurent le Cadre européen commun de référence pour les langues qui offre des lignes directrices pour l'enseignement, l'apprentissage et l'évaluation, et le Portfolio européen des langues qui permet aux apprenants à la fois d'organiser leur apprentissage et d'y réfléchir, et de mesurer et décrire leur compétence au fur et à mesure qu'ils progressent dans leur biographie d'apprentissage des langues. 
CHAREILLE - Pour une adaptation du modéle...

Le Cadre est un document qui décrit aussi complètement que possible:

$\Rightarrow$ Toutes les capacités langagières,

$\Rightarrow$ Tous les savoirs mobilisés pour les développer,

$\Rightarrow$ Toutes les situations et domaines dans lesquels on peut être amené à utiliser une langue étrangère pour communiquer.

Il est très utile aux concepteurs de programmes, aux auteurs de manuels scolaires, aux examinateurs, aux enseignants et aux formateurs d'enseignants - enfin à tous ceux concernés par l'enseignement des langues et par l'évaluation des compétences en langues. Il permet de définir, en connaissance de cause, les objectifs à atteindre lors de l'apprentissage et de l'enseignement d'une langue, et de choisir les moyens pour y parvenir.

Une récente Résolution du Conseil de l'Union européenne (novembre 2001) recommande l'utilisation de cet instrument du Conseil de l'Europe pour l'établissement de systèmes de validation de compétences en langues.

\subsection{Le Portfolio européen des langues et la notion de compétences langagières partielles.}

\subsubsection{Historique.}

Le Portfolio européen des Langues, créé en 1998, est un document dans lequel toute personne qui apprend ou a appris une langue - que ce soit à l'école ou en dehors - peut consigner ses connaissances linguistiques et ses expériences culturelles, ce qui peut l'inciter à réfléchir sur son apprentissage.

Il contient un passeport de langues que son détenteur met régulièrement à jour. Une grille lui permet de définir ses compétences linguistiques selon des critères reconnus dans tous les pays européens et de compléter ainsi les traditionnels certificats scolaires. Le document fournit aussi une biographie langagière détaillée englobant toutes les expériences faites dans les diverses langues ? cette partie est destinée à orienter l'apprenant dans la planification et l'évaluation de son apprentissage. Un dossier rassemblant des travaux personnels attestant des performances atteintes complète le tout.

\subsubsection{Buts et fonctions d'un Portfolio européen des langues.}

Le Portfolio européen des langues a deux buts principaux:

$\Rightarrow$ Motiver les apprenants en reconnaissant leurs efforts pour étendre et diversifier leurs capacités langagières à tous les niveaux.

$\Rightarrow$ Fournir un état des capacités langagières et culturelles qu'ils ont acquises (à consulter, par exemple, lorsqu'ils passent à un niveau supérieur d'apprentissage ou cherchent un emploi dans leur pays ou à l'étranger). 
et deux fonctions essentielles:

\section{$\Rightarrow$ La fonction pédagogique:}

- Accroître la motivation des apprenants pour:

- améliorer leur capacité à communiquer dans différentes langues.

- apprendre d'autres langues.

- chercher à faire de nouvelles expériences interculturelles.

- Entraîner et aider les apprenants à:

- réfléchir à leurs objectifs, à leurs manières d'apprendre et de réussir en langue étrangère.

- planifier leur apprentissage.

- apprendre de façon autonome.

- Encourager les apprenants à accroître leur expérience plurilingue et interculturelle à l'aide, par exemple :

- de contacts et de visites.

- de la lecture.

- de l'utilisation des médias.

- de réalisation de projets.

\section{$\Rightarrow$ La fonction de documentation et de présentation:}

Le Portfolio européen des langues vise à documenter la capacité langagière plurilingue et les expériences dans d'autres langues de son détenteur de manière complète, concrète, transparente et fiable. Les instruments que contient le P.E.L. aident les apprenants à faire le point sur les niveaux de compétence qu'ils ont atteints dans leur apprentissage d'une ou plusieurs langues étrangères afin de pouvoir en informer autrui de façon détaillée et comparable à un niveau international. Les occasions sont nombreuses de présenter un P.E.L. à jour, par exemple lors d'un transfert scolaire, du passage à un cycle supérieur d'enseignement, du début d'un cours de langue, de la rencontre avec un conseiller professionnel ou de la candidature à un nouveau poste. Dans tous ces cas, le Portfolio est destiné à des gens qui ont leur mot à dire dans des décisions importantes pour son propriétaire. Il se peut également qu'un apprenant soit intéressé à disposer, à titre personnel, d'une documentation de ce type.

\subsubsection{Principes.}

Toute compétence est valorisée, qu'elle ait été acquise à l'intérieur ou à l'extérieur du système d'éducation formelle :

$\Rightarrow$ Le Portfolio européen des langues est la propriété de l'apprenant.

$\Rightarrow$ Il est lié au Cadre européen commun de référence pour les langues.

$\Rightarrow$ Un ensemble de principes communs et de directives communes a été établi pour tous les Portfolios. 
CHAREILLE - Pour une adaptation du modéle...

Différents modèles de Portfolio sont/seront élaborés dans les pays membres du Conseil de l'Europe en fonction de l'âge des apprenants et des contextes nationaux, mais tous doivent respecter les règles établis et obtenir l'accréditation du Comité de Validation du Conseil de l'Europe.

Le Conseil de l'Europe a organisé en 2001 une série de séminaires pour aider les pays membres n'ayant pas participé au projet pilote à élaborer leurs modèles de Portfolio et à les mettre en oeuvre. Plusieurs O.N.G. internationales développent également des modèles de Portfolio pour l'enseignement supérieur et l'éducation des adultes.

\section{LES CENTRES DE RESSOURCES SUR LES LANGUES ÉTRANGÈRES: UN LIEU D'APPLICATION DIRECTE DES POLITIQUES LINGUISTIQUES ÉDUCATIVES EUROPÉENNES.}

\subsection{Rappel sur les fondements de l'autoformation.}

Les enjeux de la formation sont pédagogiques mais également économiques et sociaux. En effet, la modification du marché du travail implique désormais une formation à la gestion et au traitement de l'information, à la flexibilité et, de fait, à l'autoformation.

L'évolution de la société insiste actuellement sur l'adaptation de l'individu aux changements rapides apportés par la technologie. Davantage d'individus doivent être formés à des niveaux de plus en plus élevés de connaissances et de technologie. Les temps de formation et de travail ne sont plus aussi nettement dissociés qu'il y a vingt ans et l'on n'est de moins en moins sûr d'exercer le même métier dans les mêmes conditions pendant toute une carrière professionnelle. Il s'agit davantage d'acquérir des compétences pour se former tout au long de la vie que des connaissances disciplinaires ponctuelles. La valorisation sociale de la réussite individuelle, du self-made man à la mobilité géographique aisée capable de s'adapter à toute nouvelle situation vient renforcer l'image d'un homme moderne souple et évolutif.

Parallèlement le développement vertigineux des technologies de l'information et de la communication rend accessibles des quantités d'informations et de savoirs sous des formes variées. Il est encore difficile actuellement de se faire une idée de l'impact de ces phénomènes récents sur l'apprentissage proprement dit, mais il apparaît déjà comme certain que les rôles de l'école et des enseignants en ressentiront les effets. Le rapport au temps ainsi que le rapport à l'espace géographique dans les situations d'apprentissage sont questionnés par l'utilisation croissante des réseaux internet. Se pose la question de la gestion de ces modes de travail qui tend à supplanter la traditionnelle définition des contenus d'apprentissage.

Enfin, les travaux sur l'apprentissage qui se sont développés à partir du constructivisme de Jean Piaget et qui trouvent des prolongements dans les neurosciences imposent l'idée 
Cadernos de Estudos Lingüísticos 46(1) - Jan./Jun. 2004

d'un apprenant auteur de son apprentissage. Pour qu'il réussisse à produire son apprentissage, il doit être placé dans un environnement riche et stimulant dans lequel il trouvera des ressources matérielles et humaines propices à son propre développement. Son rôle dans la gestion du processus d'apprentissage sera redéfini ainsi qu'en corollaire celui de l'enseignant.

Contrairement à une représentation amplement répandue, l'apprentissage n'est pas un processus linéaire et cumulatif. Il s'agit d'une construction aléatoire par paliers successifs et instables jusqu'à la stabilisation finale qui figure la structuration de l'apprentissage. Chaque individu procède aux opérations cognitives nécessaires à la réalisation de l'apprentissage selon son propre rythme et son propre style. Le respect des caractéristiques individuelles favorise l'acquisition. Plus le public à enseigner est nombreux et divers, plus il faut éviter de le considérer comme une entité et imposer au groupe le même rythme, les mêmes types d'activités, les mêmes schémas mentaux.

Conclusion: il faut aller vers la conception de dispositifs d'autoformation (associés ou non à des cours en présentiel) permettant un maximum de liberté et d'individualisation. Ces dispositifs font nécessairement un large appel aux nouvelles technologies.

Précisons qu'un certain nombre de didacticiens parlent plus volontiers d'autonomisation, c'est-à-dire d'un processus, d'une progression vers quelque chose, que d'autonomie, c'est-à-dire d'une capacité à prendre en charge son propre apprentissage. S'il est vrai que beaucoup de termes coexistent - apprentissage autogéré, auto-enseignement, autoformation, etc.

Bien entendu, l'autonomie, que l'on peut envisager comme une fin, un but idéal ou comme une modalité d'apprentissage, pose comme la centration sur l'apprenant, d'épineux problèmes. Tout d'abord, certains apprenants, parfaitement a l'aise dans un parcours d'apprentissage balisé et transmis par l'enseignant, ne voient absolument pas l'utilité de se fatiguer à trouver leur propre chemin. D'un autre côté, tous ceux qui, pour des raisons diverses, - demandes très particulières, difficultés d'adaptation à un groupe, temps très limité par rapport à un objectif personnel, etc.- ont recours à un apprentissage autonome, n'en ont pas automatiquement les capacités car le désir ou la volonté ne sont pas suffisants pour réunir les conditions de réussite. En effet, savoir apprendre, qui en est la condition sine qua non, n'est pas a la portée de tout le monde. Finalement dans l'optique scolaire ou universitaire, où les programmes sont généralement prédéfinis et l'évaluation est essentiellement une certification, il peut sembler difficile de parler d'autonomie. Cela dit, nous pensons que l'auto-apprentissage pourrait largement contribuer à une plus grande diffusion des langues officielles car intervenant hors des systèmes éducatifs formels - tout du moins en ce qui concerne le secondaire - qui n'ont pas encore trouvé le moyen de les intégrer. Citons à ce titre l'exemple de la faculté des Lettres de l'Université Fédérale du Minas Gerais qui a créé le Centre d'auto-apprentissage de langues étrangères. Une initiative concrète pour améliorer les conditions d'enseignement et d'apprentissage de langues étrangères et pousser l'élève à développer plusieurs habilités (conversation, lecture, écrit, etc.). 
CHAREILLE - Pour une adaptation du modéle...

\subsection{Passage d'une logique de l'enseignement à une logique de l'apprentissage.}

Avant toute chose, tentons d'amorcer une réflexion préalable à la mise en place de dispositifs qui se réclament de l'auto-apprentissage et de l'autonomie de l'apprenant. En effet, les courants pédagogiques actuels qui visent au développement de tels dispositifs sont fondés sur des travaux de recherche et s'appuient sur des principes méthodologiques qu'il est préférable d'examiner avant de se lancer dans l'organisation pratique. Trop souvent les questions matérielles, accentuées actuellement par le développement galopant des T.I.C.E., occultent les questions théoriques et entravent le recours à une certaine rigueur méthodologique. Or, dans la problématique qui nous occupe - mettre l'apprenant au centre de son apprentissage en lui donnant un pouvoir réel assorti de responsabilités qui l'entraînent à devenir autonome - ne peut s'opérer sans une réflexion solide préalable à l'action pédagogique.

L'innovation pédagogique suppose la remise en cause des habitudes d'enseignement acquises dans nos cultures éducatives tels que le rôle de l'élève, le rôle de l'enseignant, l'organisation institutionnelle, l'évaluation, la représentation de l'acte d'apprendre, pour ne citer que les plus importantes.

Il convient de repenser l'organisation de l'apprentissage des langues en prenant conscience des méthodes d'enseignement et de leurs présupposés théoriques pour ensuite modifier certains paramètres en connaissance de cause. La diversité des cultures représentées devrait permettre de faire émerger l'absence de modèle d'apprentissage unique tout en pointant les convergences et les divergences. L'objectif est de proposer un modèle intégrant la prise en compte des caractéristiques individuelles de l'apprenant dans sa culture.

A ce niveau de la réflexion, soulignons que l'autoformation ne doit pas être confondue avec autodidaxie (qui s'effectue en dehors des institutions). De même, il ne s'agit pas de confondre auto-formation avec «solo-formation ». L'apprenant, agent de son propre apprentissage, bénéficie d'une orientation et de ressources. Il s'agit uniquement de former des citoyens à l'autonomie (objectif social et éducatif).

\subsection{Conséquences sur l’organisation pédagogique.}

Le passage d'une logique de l'enseignement à une logique de l'apprentissage implique que l'enseignant n'est plus seul responsable du choix des outils, des activités, du type de support, du temps et des modalités de travail. L'apprenant aura lui-même accès aux ressources mises à sa disposition. C'est lui qui est l'acteur principal de l'apprentissage une fois le dispositif organisé.

La tâche de l'enseignant consiste à sélectionner, produire et organiser les différentes ressources. Elles comprennent tous les supports existants : audio, vidéo, informatique, papier, ainsi que les enseignants, les pairs et toutes les ressources humaines disponibles. 
Cadernos de Estudos Lingüísticos 46(1) - Jan./Jun. 2004

Une organisation rigoureuse et un classement précis sont indispensables à la bonne gestion des ressources.

La production d'aides méthodologiques et d'outils de suivi des étudiants est également nécessaire au bon fonctionnement d'un tel dispositif. Est également à envisager une formation des étudiants à une manière différente d'apprendre : prendre conscience des stratégies, réfléchir aux processus d'apprentissage, s'auto-évaluer sont autant d'activités à mettre en place.

Il s'agit de rendre conscients les processus d'apprentissage des langues chez les apprenants. Il faut donner une formation psychologique et une formation technique. L'apprentissage n'est pas un produit de consommation mais à fabriquer.

\begin{tabular}{|c|l|}
\hline FORMATION PSYCHOLOGIQUE & \multicolumn{1}{c|}{ FORMATION TECHNIQUE } \\
\hline & - se regarder travailler \\
Prendre conscience que l'apprentissage & - interpréter \\
n'est pas un produit de consommation & - remettre en question ses performances \\
mais à fabrique & - s'autocorriger /s'intercorriger \\
\hline
\end{tabular}

Du côté de l'auto-évaluation, rappelons qu'elle est hautement culturelle. Il s'agit d'un ensemble d'attitudes et de compétences à développer.

$\Rightarrow$ Anticiper

$\Rightarrow$ Prendre des risques

$\Rightarrow$ Relecture

$\Rightarrow$ Etre conscient de ses résultats et prendre une décision.

Elle doit déboucher sur l'autocorrection et l'interprétation des résultats (cf. Henri HoLEc). Il s'agit de prendre en compte des critères scolaires et extra-scolaires (« recherche » de la langue hors de l'école). On peut par exemple:

$\Rightarrow$ Demander aux apprenants de s'enregistrer sur une période assez longue afin d'effectuer un travail d'analyse sur l'évolution.

$\Rightarrow$ Visionner des documents vidéos et demander aux apprenants de se fixer euxmêmes des objectifs.

$\Rightarrow$ Elaborer un carnet de bord afin d'évaluer les progrès effectués (interpréter les résultats).

$\Rightarrow$ Utiliser le système du «tandem » suivi d'une analyse de la part des apprenants.

$\Rightarrow$ Souligner les erreurs et demander aux apprenants de s'autocorriger pour, à terme, évaluer les corrections des apprenants. 
CHAREILLE - Pour une adaptation du modéle...

L'auto-évaluation doit également porter sur les stratégies d'apprentissage ce qui peut entrer dans la note finale pour valoriser l'action.

L'organisation habituelle - une classe, un enseignant, un créneau horaire - est remise en cause par un dispositif qui autorise une grande souplesse dans la gestion des flux d'étudiants, qui permet d'accueillir en un seul et même lieu des personnes de niveau différent travaillant sur des supports différents (et même des langues différentes) à des rythmes différents. Il s'agit vraiment de faire travailler les apprenants ensemble, mais en prenant en considération leurs caractéristiques individuelles tant au niveau de la motivation que des façons d'apprendre. Le danger ou le défaut de l'autoformation mal comprise réside dans l'isolement ou l'abandon de l'apprenant. S'il vient dans un centre d'autoformation au sens où nous l'entendons, c'est pour y trouver ce qu'il ne peut pas faire tout seul chez lui ou devant un ordinateur, soit des ressources nombreuses et variées, des conseils et des enseignements, des ateliers de conversation, un lieu de rencontre et de parole, en un mot un lieu de communication.

De nouveaux outils font leur apparition dans la gestion de l'enseignement / apprentissage : les fiches d'entretien-conseil, les fiches de suivi, les carnets de bord, les questionnaires sur les stratégies d'apprentissage, les activités qui entraînent à une réflexion méthodologique ? ils assurent le lien entre le travail de l'étudiant et son suivi péagogique par l'enseignant de façon à la fois personnelle et précise.

A nouveaux outils et nouvelle organisation, nouveaux enseignants ou, à tout le moins, nouvelles attitudes. Pour créer ce type de dispositif de même que pour y travailler, l'enseignant aura à développer des compétences différentes de celles auxquelles il est formé habituellement. Il devra décentrer son approche des contenus et du schéma « transmission de savoir» pour une approche axée sur l'apprenant et un schéma «acquisition de savoir ».

\subsection{L'expérience des C.R.L.}

L'objectif des Centres de Ressources sur les Langues étrangères est de stimuler l'apprenant par l'environnement. Le concept est né dans le cadre de la réflexion menée sur les compétences langagières (partielles) en vue d'une mobilité européenne annoncée, en particulier, par la création d'un Pôle universitaire européen de Strasbourg. Il répond à la question de la gestion des grands groupes hétérogènes.

Avantages présentés par les centres d'autoformation:

$\Rightarrow$ Souplesse de capacité d'accueil, c'est-à-dire un seul lieu pour:

- Des effectifs différents.

- Plusieurs matières.

- Des publics divers.

- Une grande variété de supports d'apprentissage. 
$\Rightarrow$ Favorise: les relations pédagogiques personnalisées.

- Le travail en équipe des apprenants.

$\Rightarrow$ Développe des compétences d'autonomie chez les apprenants.

Les centres de ressources sur les langues développent :

$\Rightarrow$ Les compétences linguistiques et culturelles.

$\Rightarrow$ Une capacité à apprendre les langues.

$\Rightarrow$ Une capacité à appréhender des cultures différentes.

$\Rightarrow$ La médiation culturelle.

$\Rightarrow$ La socialisation et la citoyenneté (européenne) de l'individu.

Typologie des centres d'autoformation:

$\Rightarrow$ Le cours et le centre sont interdépendants.

$\Rightarrow$ Le centre est indépendant des cours.

$\Rightarrow$ Tout se passe dans le centre.

$\Rightarrow$ Autre.

$\Rightarrow$ Un seul / plusieurs lieu(x).

Un Centre de Ressources de Langues propose des ressources variées pour l'apprentissage des langues. Les étudiants viennent y travailler selon leurs objectifs et ceux de l'institution. Ils font partie d'un groupe constitué dans leur discipline, sans critère de niveau, qu'un enseignant encadre dans un créneau horaire fixe. Cet accès fixe peut être complété par un accès libre dans des créneaux prévus à cet effet.

Il comprend cinq espaces de travail:

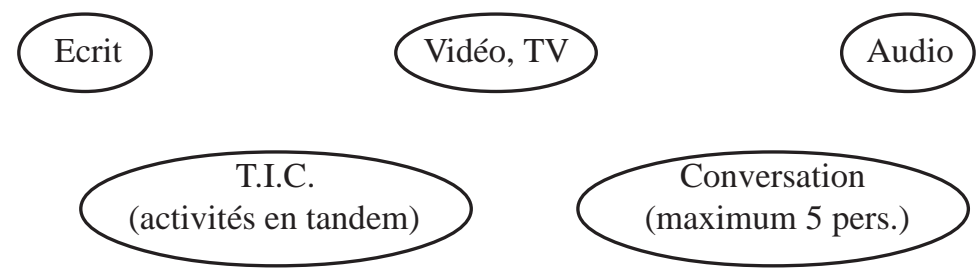

Les centres utilisent le modèle du Portfolio européen des langues permettant une meilleure gestion des parcours d'apprentissage par les étudiants et une valorisation des compétences acquises.

L'apprentissage des langues est organisé en modules, gradués en fonction des niveaux du Cadre européen commun de référence du Conseil de l'Europe : niveaux A1, A2, B1, B2, $\mathrm{C} 1$ et $\mathrm{C} 2$. Les niveaux B1, B2 et C1 correspondent respectivement aux degrés 1, 2 et 3 de la Certification en Langues de l'Enseignement Supérieur (C.L.E.S.). A chaque niveau correspondent certaines tâches à réaliser. 
Les étudiants peuvent profiter de l'aide d'un tuteur (enseignant de langue) en ligne. Ils sont affectés à un groupe et sont encouragés à collaborer entre eux. La communication se fait par courrier électronique, chat et forum de discussion. Le tuteur propose un programme de travail et un calendrier pour les discussions en groupe sur le réseau. Les étudiants remplissent un carnet de bord en ligne, dans lequel sont régulièrement notés le travail effectué et le temps consacré.

L'enseignant conseille et informe les étudiants, notamment en ce qui concerne les objectifs d'apprentissage. A chaque séance de travail, les étudiants remplissent une feuille de suivi, sur laquelle ils notent la référence des ressources utilisées, le travail effectué et sa durée. Cette trace écrite permet de faire régulièrement le point sur le travail accompli.

Il apparaît qu'un centre de ressources de langues ne peut réussir qu'à condition d'être :

$\Rightarrow$ Le résultat d'un projet pédagogique préalable et collectif, porté par l'institution, confié à une équipe de mise en oeuvre motivée et compétente.

$\Rightarrow$ Doté des moyens nécessaires - enseignants, moniteurs, personnel administratif et technique -, en temps de production pédagogique préalable, en locaux, en matériel.

$\Rightarrow$ En prise sur l'évolution technologique et à même de maîtriser les problèmes de réseaux.

Côté enseignants, l'expérience montre que ces derniers ne sont motivés pour produire et partager des dossiers plurimédias d'auto-apprentissage que dans la mesure où ce travail est pris en compte dans leur service, leur carrière rémunéré sur des bases claires.

Cette production, par ailleurs doit être le fruit d'un travail d'équipe, et être validée en interne, sous peine d'usure de la créativité, de non-pertinence par rapport aux objectifs, de qualité finale incertaine. Il est donc nécessaire de trouver des financements, de mutualiser les moyens internes et les productions centre-centres de langues.

Enfin il est nécessaire que les enseignants soient d'accord sur leur nouveau rôle de facilitateur / accompagnateur, qu'ils soient formés à la culture éducative, qu'ils fassent confiance aux apprenants, et acceptent de travailler en équipe.

Côté étudiants, l'ensemble ne pourra marcher que dans la mesure où l'accès au centre d'auto-apprentissage sera gratuit, et où leur travail sera validé en fin de parcours. Ce parcours, suite à une évaluation initiale, sera défini sur la base de compétences à acquérir. Il sera facilité par un conseil-guidage et la possibilité permanente d'auto-évaluer ses progrès.

\section{BIBLIOGRAPHIE}

C.E.P.A.L. (1995). «Los bachilleres uruguayos: quienes son, qué aprendieron y qué opinan» in Boletín: proyecto principal de educación $\mathrm{n}^{\circ} 37$, Santiago de Chile-Chile, p.p. 35-52. 
CHAREILLE, Samantha. (juin 2002). « Deux cents ans d'enseignement du français en Colombie » in Travaux de didactique du français langue étrangère $\mathrm{n}^{\circ}$ 45, I.E.F.E., Université Paul Valéry-Montpellier III (Eds.), Montpellier, $22 \mathrm{p}$.

CHAREILLE, Samantha. (juin 2003). «Histoire de la diffusion de la langue française en Uruguay depuis le $\mathrm{XIX}^{\mathrm{e}}$ siècle » in Sudlangues n², Faculté des Lettres et Sciences Humaines de l'Université Cheikh Anta Diop de Dakar, $24 \mathrm{p}$.

CHAREILLE, Samantha. (january 2003). «Planificación lingüística y constitución de un bloque regional: el caso del Mercosur (Argentina, Brasil, Paraguay y Uruguay) y de Chile » in Language problems and laguage planning, volume n²7, John Benjamin publishing company, Amsterdam, The Netherlands, 8 p.

CHAREILLE, Samantha. (décembre 2002). «Entre formations linguistiques et économie » in Sudlangues $\mathrm{n}^{\circ} 1$, Faculté des Lettres et Sciences Humaines de l'Université Cheikh Anta Diop de Dakar, 12 p.

CHAREILLE, Samantha (sous la direction de Daniel Coste). (mai 2001). Aménagement linguistique et constitution d'un ensemble régional : le cas du Mercosur (Argentine, Brésil, Paraguay et Uruguay) et du Chili, Mémoire de Thèse de Doctorat de didactologie des langues et des cultures, deux volumes, U.F.R. de Didactique du F.L.E., Université PARIS III - Ecole normale supérieure de Lettres et Sciences humaines de Lyon, Paris.

CHAREILLE, Samantha (sous la direction d'Elisabeth GuimBRETIÈRE). (juin 1997). Statut du français en Uruguay à la fin des années quatre-vingt-dix : raisons du déclin, Mémoire de D.E.A. de langage en situation option « situation d'acquisition et d'apprentissage », U.F.R. D.E.S.C.I.L.A.C., Université de Mont-SaintAignan, Rouen.

DABENE, Louise. (1994). Repères sociolinguistiques pour l'enseignement des langues, Hachette-F.L.E, collection F-Références, Baume-les-Dames.

I A Assemblée générale de la Conférence parlementaire des Amériques, 18-22 septembre 1997, «Education et formation: entre l'incontournable compétition et l'indispensable égalité des chances», Québec, Canada.

La Nación, 12 février 1988, Buenos Aires, Argentine.

TOFFLER, Alvin. (1987). Choc du Futur, Folios Essais, Paris.

U.N.I.C.E.F. (1999). La situation des enfants dans le monde 1999: Education, extrait du site Internet de l'U.N.I.C.E.F.: www.unicef.org/french.

U.N.I.C.E.F. (2000). La situation des enfants dans le monde 2000, extrait du site Internet de l'U.N.I.C.E.F.: www.unicef.org/french. 